\title{
Prevalencia de Bruxismo del Sueño en Niños y su Relación con los Signos de Trastornos Temporo- mandibulares y las Parafunciones Diurnas
}

\author{
Prevalence of Sleep Bruxism in Children, and its Relationship with \\ Signs of Temporomandibular Disorders and Daytime Oral Parafunctions
}

Héctor Sandoval Ulloa* \& María Paz Fariña Vélez

SANDOVAL, U. H. \& FARIÑA, V. M. P. Prevalencia de bruxismo del sueño en niños y su relación con los signos de trastornos temporomandibulares y las parafunciones diurnas. Int. J. Odontostomat., 10(1):41-47, 2016.

RESUMEN: El bruxismo del sueño (BS), es el acto de apretar y rechinar los dientes mientras se duerme, lo cual puede producir serias consecuencias en los niños, como el desgaste dentario y dolor muscular. El objetivo de esta investigación fue determinar la prevalencia del BS en niños, además de relacionarlo con la presencia de signos de trastornos temporomandibular (TTM) y de las parafunciones diurnas (PFD). La muestra está conformada por 369 estudiantes de 6 a 14 años. El diagnóstico de BS se basa en los criterios de inclusión de la Academia Americana de Medicina del Sueño, con reporte de los padres indicando la historia nocturna de apriete/rechinamiento dentario, presencia de PFD y antecedentes médicos. La información se complementó con examen clínico de signos de TTM y desgaste dentario. Para análisis estadístico se utilizaron las pruebas Chi-Cuadrado y Odds Ratio, con un intervalo de confianza de $95 \%$. La prevalencia de BS fue de $32 \%$. Los niños de 6 años presentan la mayor prevalencia (38\%), y los de 14 años la menor (27\%). De los individuos con BS, el $77 \%$ presentaban signos de TTM $(p<0,0001 ;$ OR: 5,4$)$ y el $87 \%$ presentaba PFD $(p=0,0003 ;$ OR= 2,9). La prevalencia de BS, su distribución por edad y sexo, se corresponde con la evidencia científica internacional. El BS es un factor de riesgo para los TTM, aumentando las probabilidades de que las personas puedan sufrir mialgias o artralgias. En las parafunciones, el hábito de morder durante el día es común en los pacientes con BS. El tratamiento llevado a cabo durante la infancia con un diagnóstico temprano, podría ser capaz de prevenir el daño a la salud de un individuo como futuro adulto.

PALABRAS CLAVE: prevalencia, bruxismo del sueño, niños, trastornos de la articulación temporomandibular, parafunciones.

\section{INTRODUCCIÓN}

A la fecha, el Bruxismo del Sueño (BS) ha sido definido por la Academia Americana de Medicina del Sueño (AASM) como "una actividad muscular mandibular caracterizada por apretar o rechinar de los dientes y/o por refuerzos o empuje de la mandíbula durante el sueño". Los pacientes pueden experimentar dolor muscular o de cabeza por la mañana, pudiendo desgastar el esmalte de los dientes (American Academy of Sleep Medicine, 2014). Si bien se puede presentar a cualquier edad, la prevalencia en niños fluctúa entre un $3,5 \%$ hasta un $40,6 \%$, no tiene predilección por sexo y va disminuyendo con la edad (Manfredini et al., 2013; Varalakshmi Reddy et al., 2014).
El BS puede ocasionar serias consecuencias en los niños no sólo relacionadas con el desgaste dentario, fatiga o dolor, sino que también afecta la calidad del sueño, durmiendo menos horas de las recomendadas para su edad y altera el descanso (SimõesZenari \& Bitar, 2010), provocando una disminución en su rendimiento académico (Lam et al., 2011).

EI BS presenta una etiología multifactorial, involucrando procesos fisiológicos multisistémicos muy complejos (Klasser et al., 2015). Debido a esto, se ha dividido en dos grandes clasificaciones: BS primario o idiopático del sueño, cuando no hay causas claras identificables, y BS Secundario, en el que sí está aso-

* Cirujano - Dentista. Universidad de Concepción, Concepción, Chile.

** Especialista en TTM y Dolor Orofacial, Universidad de Valparaiso, Valparaiso, Chile. 
ciado a una condición socio-psicológica o médica, como los trastornos respiratorios del sueño, problemas neurológicos o condiciones psiquiátricas y el consumo de drogas o medicamentos (Lavigne et al., 2005; Kato \& Lavigne, 2010; Lobbezoo et al., 2013).

La evidencia científica demuestra que además se debe poner énfasis en los signos y síntomas padecidos por los pacientes con BS, tales como: dolor o molestias en la cara durante la mañana, dolor de cabeza, desgaste dentario, hipertrofia y/o dolor de los músculos masticatorios y la presencia de ruidos articulares de la ATM a la palpación (Machado et al., 2014)

Trastornos de la Articulación Temporomandibular (TTM). A nivel de la Articulación Temporomandibular (ATM), por lo general los niños con BS presentan baja sintomatología. Aun así, se ha descrito que los niños con BS presentan una mayor predisposición a tener trastornos temporomandibulares que los niños sin BS (Restrepo et al., 2008) describiendo al BS como un factor de riesgo doloroso (Fernandes et al., 2012) Además, los niños con historial de apriete/rechinamiento dentario reportaban más sonidos en su ATM y mayor fatiga muscular en comparación con niños sin bruxismo (Carra et al., 2011).

Parafunciones Diurnas (PFD). Actividades como morderse las uñas, labios o mejillas han sido ampliamente descritas como predisponentes de BS (Carlsson et al., 2003) lo mismo para morder objetos o lápices (Serra-Negra et al., 2012; Vieira-Andrade et al., 2014) y el hábito de masticar chicle (Cortese et al., 2013). De todos estos, se ha descrito que el más común es morderse las uñas (Farsi, 2003). Los autores coinciden en que el hábito de morder en vigilia es un factor común en éstos pacientes lo que podría desarrollar la parafunción nocturna. En el caso de los niños que mordían sus labios, estos tienen 5 veces más posibilidades de tener BS (Simões-Zenari \& Bitar).

El objetivo de esta investigación fue determinar la prevalencia de BS en niños de la localidad de Los Muermos, región de Los Lagos, y relacionarlo con los signos de TTM y PFD.

\section{MATERIAL Y MÉTODO}

Selección de la Muestra: Se revisó a un total de 369 estudiantes de enseñanza básica de las escuelas urbanas de la comuna de Los Muermos, Región de Los
Lagos. Todos los padres de los participantes firmaron debidamente el consentimiento informado. Esta investigación se encuentra autorizada por el Comité de bioética de la Facultad de Odontología de la Universidad de Concepción.

Métodos y técnicas de investigación: Se desarrollaron dos instrumentos de recolección de datos. Uno corresponde a un cuestionario respondido por los padres, acerca de la historia nocturna de apriete/ rechinamiento dentario, la presencia de PFD (Morderse las uñas, objetos como lápices, los labios, las mejillas y masticar chicle) y su historia médica. Los padres fueron instruidos tanto en la observación de sus hijos, como en el registro del cuestionario en sí. El segundo corresponde a una ficha clínica, en la cual se registraron los signos de BS y de TTM observados en los niños durante el examen clínico. Solo un examinador realizó el examen clínico, para uniformar el diagnóstico.

Diagnóstico de Bruxismo del Sueño. El diagnóstico de Bruxismo del Sueño fue determinado mediante el Cuestionario de los Padres, junto al examen clínico.

Se utilizaron los criterios de inclusión propuesto por la Academia Americana de Medicina del Sueño, el cual incluye (American Academy of Sleep Medicine; Grigg-Damberger, 2012):

- Reporte de los padres que indican la ocurrencia de rechinar de dientes audible en la noche.

- Que no exista administración de medicamentos.

- No exista algún desorden médico o mental (ej. Epilepsia relativa al sueño).

- Que no hayan otros desordenes del sueño. (ej. Apnea obstructiva del sueño).

Diagnóstico de Signos de TTM. El diagnóstico fue realizado mediante examen clínico, utilizando los Criterios Diagnósticos para Trastornos Temporomandibulares (DC/TMD) (Restrepo et al., 2008) el cual incluye la evaluación Clínica de:

- Dolor en Músculos Masticatorios en reposo o durante la función mandibular.

- Sonidos en la ATM: Chasquido o crepitación.

- Dolor en la ATM a la palpación.

Análisis e interpretación de los datos. Los datos recopilados se tabularon mediante el programa computacional Microsoft Excel y fueron analizados en programa computacional Infostat. Se comenzó con análisis descriptivo univariado de la variable BS, para 
luego realizar el análisis bivariado con las variables de interés (TTM y PFD). Para analizar la asociación se utilizaron las pruebas Chi-Cuadrado de independencia y para cuantificar la asociación el Odds Ratio, con un intervalo de confianza de $95 \%$.

\section{RESULTADOS}

La muestra estuvo conformada por 369 individuos, 158 hombres (43\%) y 211 mujeres (57\%), con edades entre los 6 y los 14 años. La prevalencia de bruxismo del sueño resultante fue de 118 niños (32 $\%$ ), 51 hombres (32\% del total de hombres) y $67 \mathrm{mu}-$ jeres $(31 \%$ del total de mujeres) $(p=0,91)$ (Tabla I). Por edad, la mayor prevalencia se observó a los seis años $(38 \%)$, y la menor a los 14 años $(27 \%)(p=0,94)$ (Fig. 1).
En cuanto a la frecuencia de los episodios de BS, según reporte de los padres, un $31 \%$ de los niños apretaba sus dientes solo una vez por semana, un 48 $\%$ lo hacía de una a tres veces por semana y un $21 \%$ más de cuatro veces por semana (Tabla II).

De los niños con BS, 91 (77\%) presentaron algún TTM, mientras que 27 (23 \%) no lo manifestaron $(p<0,0001 ; O R=5,4)$ (Tabla III). El TTM más frecuente en los individuos con BS fue el dolor del músculo Masetero que se encontraba en un $66 \%$ de los individuos, seguido del dolor de la ATM (58\%), dolor del músculo Temporal $(40 \%)$ y finalmente los ruidos articulares (7\%) (Fig. 2).

Al comparar la presencia de PFD en niños con BS, 103 niños (87 \%) presentaban PFD, mientras que sólo $15(13 \%)$ no presentaban PFD $(p=0,0003$; OR= 2.9) (Tabla IV). La PFD más frecuente fue morder ob-

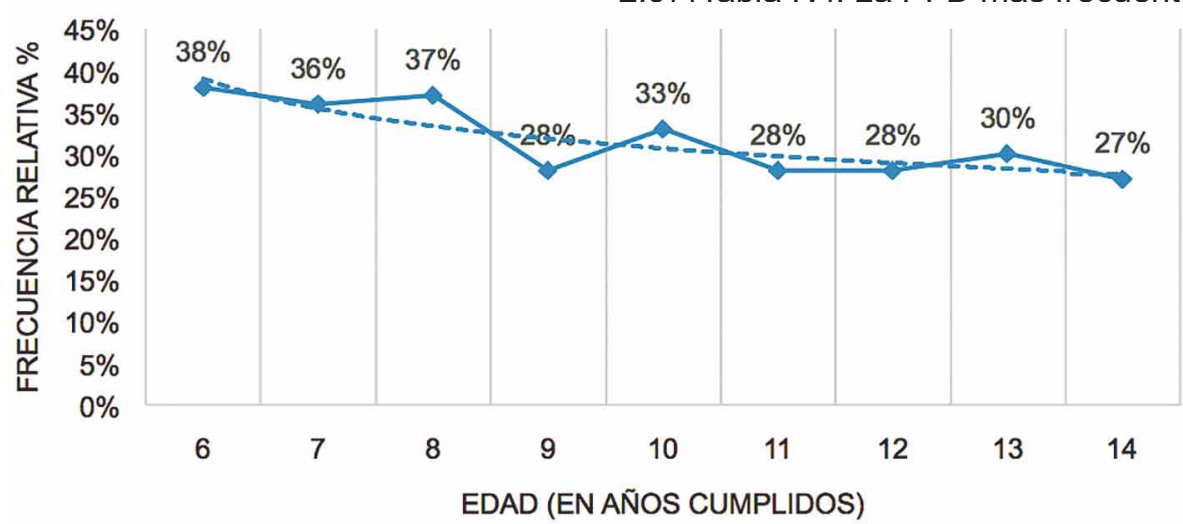

Fig 1. Prevalencia de Bruxismo del Sueño por edad.

Tabla I. Prevalencia de Bruxismo del Sueño.

\begin{tabular}{lcccc}
\hline Prevalencia de BS & Hombres & Mujeres & Total & $\%$ \\
\hline Con diagnóstico de BS & 51 & 67 & 118 & $32 \%$ \\
Sin Diagnóstico de BS & 107 & 144 & 251 & $68 \%$ \\
\hline
\end{tabular}

Tabla II. Frecuencia de eventos de Bruxismo del Sueño.

\begin{tabular}{ccc}
\hline $1 \mathrm{vez}$ & $\begin{array}{c}\text { Frecuencia por semana } \\
\text { De } 1 \mathrm{a} 3 \text { veces }\end{array}$ & Más de 4 veces \\
\hline $31 \%$ & $48 \%$ & $21 \%$ \\
\hline
\end{tabular}

Tabla III. Cruce de variables "Bruxismo del Sueño" con "Diagnóstico de Signos de Trastornos Temporomandibulares".

\begin{tabular}{lcc}
\hline & \multicolumn{2}{c}{$\begin{array}{c}\text { Diagnóstico de Signos de Trastornos } \\
\text { Temporomandibulares (TTM) }\end{array}$} \\
\cline { 2 - 3 } & Presente & Ausente \\
\hline Con Bruxismo del Sueño & $77 \%$ & $23 \%$ \\
Sin Bruxismo del Sueño & $39 \%$ & $61 \%$ \\
\hline
\end{tabular}


jetos (59\%), seguido de morderse las uñas (54\%), masticar chile $(49 \%)$ y finalmente morderse las mejillas y/o los labios (36 \%) (Fig. 3).

Tabla IV. Cruce de variables "Bruxismo del Sueño" - "Presencia de Parafunciones Diurnas".

\begin{tabular}{lcc}
\hline & \multicolumn{2}{c}{ Parafunciones Diurnas (PFD) } \\
\cline { 2 - 3 } & Presencia & Ausencia \\
\hline Con Bruxismo del Sueño & $87 \%$ & $13 \%$ \\
Sin Bruxismo del Sueño & $70 \%$ & $30 \%$ \\
\hline
\end{tabular}

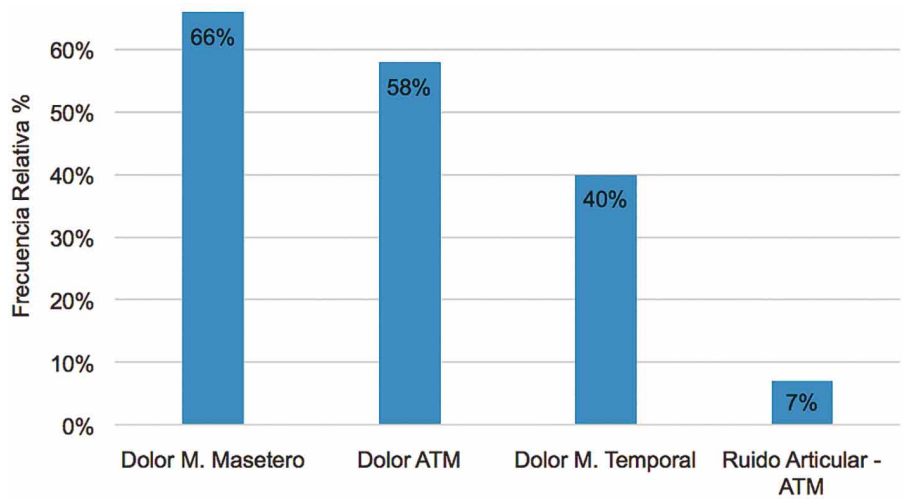

Fig 2. Signos de Trastornos Temporomandibulares más frecuentes en individuos con Bruxismo del Sueño.

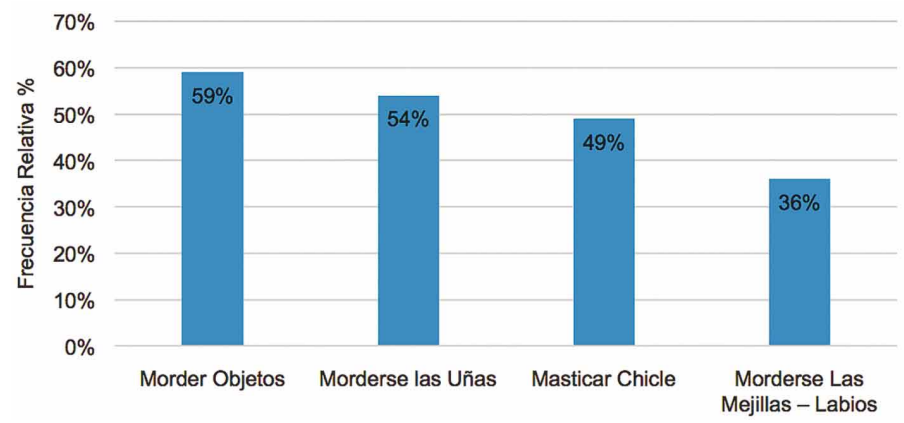

Fig. 3. Parafunciones Diurnas más frecuentes en individuos con diagnóstico de Bruxismo del Sueño.

\section{DISCUSIÓN}

La prevalencia de BS en el presente estudio corrobora la evidencia científica, con una prevalencia de un 32 $\%$. Se observa que la prevalencia disminuye con la edad, partiendo con un $38 \%$ a los seis años, hasta llegar a un 27 $\%$ a los 14 años $(p=0,94)$, estableciendo que las variables son independiente estadísticamente.
Al revisar estudios con metodologías similares, se encuentran varios casos de Brasil que muestran diferentes prevalencias de bruxismo del sueño. En un estudio realizado en Bello Horizonte se pueden apreciar resultados similares, con una prevalencia de $35,3 \%$ en una muestra de 652 niños (Serra-Negra et al. 2009, 2010). En otro estudio con metodología similar, se encontró una prevalencia de BS de un $15 \%$, en una muestra de 170 niños (Fonseca et al., 2011). Castelo et al. (2005) reportó una prevalencia de un $32 \%$. En Canadá también se han realizado estudios similares, con cuestionario para los padres y examen clínico de los niños encontrando una prevalencia de $15 \%$ con una muestra de 604 niños de 7 a 17 años, los cuales tendían a tener mayor riesgo de experimentar algún dolor o fatiga muscular junto con dolor de cabeza. En este estudio la mayor prevalencia de BS la tenían los niños menores de 12 años (Carra et al.). En Hong Kong se encontró una prevalencia de $6 \%$ en niños (Lam et al.). En Kentucky, EEUU, se encontró una prevalencia de $36,8 \%$ en individuos de seis años (Insana et al., 2013).

Distintas revisiones bibliográficas han establecido un rango para la prevalencia de BS, partiendo por una que el 2013 proponía un rango desde un 3,5-40,6 \% (Manfredini et al., 2013), y otra más actual del 2014, un rango de 5,9-49,6\% (Machado et al.).

En cuanto a la prevalencia del BS por sexo, en el presente estudio se encuentra que dentro de los hombres, un $32 \%$ de ellos tiene BS, y en las mujeres, un $31 \%$ de ellas $(p=0,91)$, no existiendo relación estadística entre ambas, por lo que las variables son independientes. Algunos estudios han mostrado diferencias entre sexo, con una prevalencia mayor en mujeres (Castelo et al.) y otros con mayor prevalencia en hombres (Lam et al.; Insana et al.). Sin embargo, en la gran mayoría de investigaciones no se han encontrado diferencias estadísticas significativas entre hombres y mujeres (Manfredini et al., 2013; Carra et al.; Serra-Negra et al., 2009; Fonseca et al.; Lavigne et al., 2011). Respecto a la edad, se ha encontrado que la prevalencia de BS disminuye con el pasar de los años (Manfredini et al., 2013; SerraNegra et al., 2009; Lam et al.; Lavigne et al., 2011). 
En adultos con BS, más del $86 \%$ de ellos han reportado tener BS en su infancia, por lo que el bruxismo del sueño en la infancia puede persistir en la vida adulta (Hublin \& Kaprio, 2003; Carlsson et al.). Cuando los síntomas de BS persisten con el pasar de los años, podría tener mayores efectos perjudiciales en las estructuras orofaciales (Nowak \& Warren, 2000).

Diagnóstico de Bruxismo del Sueño. La metodología para determinar la presencia o ausencia de BS a través del reporte de padres por el sonido generado durante el rechinamiento/apriete dentario ha sido utilizado ampliamente en los distintos estudios de prevalencia de BS (Carra et al.; Serra-Negra et al., 2009, 2010; Fonseca et al.; Castelo et al.; Lavigne et al., 2011). Durante el examen clínico, solo la presencia de desgaste dentario no debe asociarse absolutamente con diagnóstico de BS (Abe et al., 2009), a pesar de que el BS es un factor de riesgo significante, ya que no es el único capaz de producir desgastes dentarios, por lo tanto no puede indicar por sí solo la ocurrencia actual de BS. Sujetos que han tenido BS en el pasado, también pueden presentar estas facetas de desgaste incluso si el hábito ya no existe, y por otro lado, los sujetos que han comenzado recientemente a mostrar BS no muestran signos de desgaste tan rápidamente (Castelo et al.). Esta limitación se ve superada con el reporte de los padres del sonido de rechinar/ apretar los dientes, que sirve de guía para el diagnóstico de BS. Otro factor que podría contribuir a la subestimación de la presencia de BS es que la mayoría de los niños no duermen en habitaciones de sus padres o cerca de ellos (Quintero et al., 2009).

Por lo tanto, se debe ser cuidadoso ya que los niños en general son inconscientes de este hábito (Castelo et al.; Bader \& Lavigne, 2000), y del mismo modo, las Parafunciones diurnas podrían no ser reveladas durante una consulta dental de rutina (SerraNegra et al., 2009; Castelo et al.).

Bruxismo del Sueño y Trastornos Temporomandibulares.. En este estudio encontramos que el diagnóstico de BS es estadísticamente dependiente del diagnóstico de TTM $(p<0,0001)$. Además, pacientes con BS tienen cinco veces más posibilidades de padecer algún TTM, comparado con los pacientes sanos $(O R=5.4)$. Dentro de los TTM que sufren los niños con BS, el más frecuente es el dolor del músculo Masetero.

Estos hallazgos concuerdan con la evidencia científica, ya que se ha establecido una asociación previa entre el BS como un signo o causa de TTM tanto en la población pediátrica (Pereira et al., 2010) como en la población adulta (Fernandes et al.), sobretodo en el papel que tiene el BS sobre el componente musculo esquelético, con dolor (mialgia) o fatiga muscular (Fernandes et al.; Carra et al.), estos resultados contribuyen en la hipótesis de que el BS podría participar en la predisposición, iniciación y perpetuación de los TTM (Fernandes et al.).

Es importante destacar que en los distintos estudios, el BS ha sido encontrado con frecuencia en los pacientes con condiciones dolorosas de TTM, en mialgias y artralgia (Manfredini et al., 2003).

Bruxismo del Sueño y Parafunciones Diurnas. Los pacientes con BS también presentan una asociación estadísticamente dependiente con la presencia de Parafunciones $(p=0,0003)$, con tres veces más posibilidades de tener una parafunción diurna, en comparación con los individuos sin $\mathrm{BS}(\mathrm{OR}=2,9)$.

Las parafunciones más frecuentes en los individuos con BS son el acto de morder objetos y morderse las uñas, hecho que se correlaciona con la evidencia científica, ya que se ha establecido ambas son las parafunciones diurnas más frecuentes (Farsi).

Para finalizar, se ha descrito que los individuos que presentan en conjunto la parafunción nocturna de BS y las parafunciones diurnas tienden a tener más signos y síntomas de TTM (Carra et al.).

Por lo tanto, el tratamiento llevado a cabo durante la infancia con un diagnóstico temprano, podría ser capaz de prevenir el daño a la salud de un individuo como futuro adulto, sobre todo en el componente muscular. Los profesionales de la salud, incluidos los odontólogos, deben ser alentados a asesorar a las familias sobre la importancia del BS y las consecuencias que podría provocar a futuro.

SANDOVAL, U. H. \& FARIÑA, V. M. P. Prevalence of sleep bruxism in children, and its relationship with signs of temporomandibular disorders and oral parafunctions. Int. J. Odontostomat., 10(1):41-47, 2016.

ABSTRACT: Sleep Bruxism (SB) is the act of grinding and clenching teeth during sleep, which could lead serious consequences in children, such a tooth wear and muscle pain. The objective of this research was to determine the prevalence of SB in children, and to relate the presence of signs of temporomandibular disorders (TMD) and daytime 
oral parafunctions. The sample consists of 369 students of 6-14 years old. SB diagnosis is based on the inclusion criteria of the American Academy of Sleep Medicine, with parent report indicating the history of tooth clenching/grinding, presence of awake oral parafunctions and medical history. The information was complemented by clinical examination of signs of TMD and tooth wear. For statistical analysis chisquare test and Odds Ratio Test was used, with a confidence interval of $95 \%$. The prevalence of SB was $32 \%$. Children 6 years old had the highest prevalence (38\%), and children 14 years old had less (27\%). Individuals with SB, $77 \%$ showed signs of TMD $(p<0.0001 ; O R=5.4)$ and $87 \%$ had daytime parafunctions $(p=0.0003 ; O R=2.9)$. The prevalence of SB, its distribution by age and sex, corresponds to the international scientific evidence. The SB is a risk factor for TMD, increasing the probabilities that people may suffer muscle or joint aches. Daytime parafunctions are common in patients with SB. The treatment carried out during childhood with an early diagnosis, might be able to prevent damage to the health of an individual as a future adult.

KEY WORDS: prevalence, sleep bruxism, children, temporomandibular joint disorders, parafunctions.

\section{REFERENCIAS BIBLIOGRÁFICAS}

Abe, S.; Yamaguchi, T.; Rompré, P. H.; De Grandmont, P.; Chen, Y. J. \& Lavigne, G. J. Tooth wear in young subjects: a discriminator between sleep bruxers and controls? Int. J. Prosthodont., 22(4):342-50, 2009.

American Academy of Sleep Medicine. The International Classification Of Sleep Disorders, Revised: Diagnostic and Coding Manual. 3rd ed. Westchester, American Academy of Sleep Medicine, 2014.

Bader, G. \& Lavigne, G. Sleep bruxism; an overview of an oromandibular sleep movement disorder. Sleep Med. Rev., 4(1):27-43, 2000.

Carlsson, G. E.; Egermark, I. \& Magnusson, T. Predictors of bruxism, other oral parafunctions, and tooth wear over a 20-year follow-up period. J. Orofac. Pain, 17(1):50-7, 2003.

Carra, M. C.; Huynh, N.; Morton, P.; Rompré, P. H.; Papadakis, A.; Remise, C. \& Lavigne, G. J. Prevalence and risk factors of sleep bruxism and wake-time tooth clenching in a 7- to 17-yr-old population. Eur. J. Oral Sci., 119(5):386-94, 2011.

Castelo, P. M.; Gavião, M. B.; Pereira, L. J. \& Bonjardim, L. R. Relationship between oral parafunctional/nutritive sucking habits and temporomandibular joint dysfunction in primary dentition. Int. J. Paediatr. Dent., 15(1):29-36, 2005.
Cortese, S. G.; Fridman, D. E.; Farah, C. L.; Bielsa, F.; Grinberg, J. \& Biondi, A. M. Frequency of oral habits, dysfunctions, and personality traits in bruxing and nonbruxing children: a comparative study. Cranio, 31(4):283-90, 2013.

Farsi, N. M. Symptoms and signs of temporomandibular disorders and oral parafunctions among Saudi children. J. Oral Rehabil., 30(12):1200-8, 2003.

Fernandes, G.; Fanco, A. L.; Siqueira, J. T.; Gonçalves, D. A. \& Camparis, C. M. Sleep bruxism increases the risk for painful temporomandibular disorder, depression and non-specific physical symptoms. J. Oral Rehabil., 39(7):538-44, 2012.

Fonseca, C. M.; dos Santos, M. B.; Consani, R. L.; dos Santos, J. F. \& Marchini, L. Incidence of sleep bruxism among children in Itanhandu, Brazil. Sleep Breath, 15(2):21520, 2011.

Grigg-Damberger, M. M. The AASM Scoring Manual four years later. J. Clin. Sleep Med., 8(3):323-32, 2012.

Hublin, C. \& Kaprio, J. Genetic aspects and genetic epidemiology of parasomnias. Sleep Med. Rev., 7(5):41321, 2003.

Insana, S. P.; Gozal, D.; McNeil, D. W. \& Montgomery-Downs, H. E. Community based study of sleep bruxism during early childhood. Sleep Med., 14(2):183-8, 2013.

Kato, T. \& Lavigne, G. J. Sleep bruxism: A sleep-related movement disorder. Sleep Med. Clin., 5(1):9-35, 2010.

Klasser, G. D.; Rei, N. \& Lavigne, G. J. Sleep bruxism etiology: the evolution of a changing paradigm. J. Can. Dent. Assoc., 81:f2, 2015.

Lam, M. H.; Zhang, J.; Li, A. M. \& Wing, Y. K. A community study of sleep bruxism in Hong Kong children: association with comorbid sleep disorders and neurobehavioral consequences. Sleep Med., 12(7):641-5, 2011.

Lavigne, G. J.; Manzini, C. \& Huynh, N. T. Sleep Bruxism. In: Kryger, M. H.; Roth, T. \& Dement, W. C. (Eds.). Principles and Practice of Sleep Medicine. St. Louis, Elsevier Saunders, 2011. pp.1129-39.

Lavigne, G. J.; Manzini, C. \& Kato, T. Sleep Bruxism. In: Kryger, M. H.; Roth, T. \& Dement, W. C. (Eds.). Principles and Practice of Sleep Medicine. 4th ed. Philadelphia, Elsevier Saunders, 2005. pp.946-59.

Lobbezoo, F.; Ahlberg, J.; Glaros, A. G.; Kato, T.; Koyano, K.; Lavigne, G. J.; de Leeuw, R.; Manfredini, D.; Svensson, P. \& Winocur, E. Bruxism defined and graded: an international consensus. J. Oral Rehabil., 40(1):2-4, 2013. 
Machado, E.; Del-Fabbro, C.; Cunali, P. A. \& Kaizer, O. B. Prevalence of sleep bruxism in children: a systematic review. Dental Press J. Orthod., 19(6):54-61, 2014.

Manfredini, D.; Cantini, E.; Romagnoli, M. \& Bosco, M. Prevalence of bruxism in patients with different research diagnostic criteria for temporomandibular disorders (RDC/TMD) diagnoses. Cranio, 21(4):279$85,2003$.

Manfredini, D.; Restrepo, C.; Diaz-Serrano, K.; Winocur, E. \& Lobbezoo, F. Prevalence of sleep bruxism in children: a systematic review of the literature. J. Oral Rehabil., 40(8):631-42, 2013.

Nowak, A. J. \& Warren, J. J. Infant oral health and oral habits. Pediatr. Clin. North Am., 47(5):1043-66, 2000.

Pereira, L. J.; Pereira-Cenci, T.; Del Bel Cury, A. A.; Pereira, S. M.; Pereira, A. C.; Ambosano, G. M. \& Gavião, M. B. Risk indicators of temporomandibular disorder incidences in early adolescence. Pediatr. Dent., 32(4):324-8, 2010.

Quintero, Y.; Restrepo, C. C.; Tamayo, V.; Tamayo, M.; Vélez, A. L.; Gallego, G. \& Peláez-Vargas, A. Effect of awareness through movement on the head posture of bruxist children. J. Oral Rehabil., 36(1):16-25, 2009.

Restrepo, C. C.; Vásquez, L. M.; Alvarez, M. \& Valencia, I. Personality traits and temporomandibular disorders in a group of children with bruxing behaviour. J. Oral Rehabil., 35(8):585-93, 2008.

Serra-Negra, J. M.; Paiva, S. M.; Auad, S. M.; RamosJorge, M. L. \& Pordeus, I. A. Signs, symptoms, parafunctions and associated factors of parentreported sleep bruxism in children: a case-control study. Braz. Dent. J., 23(6):746-52, 2012.

Serra-Negra, J. M.; Paiva, S. M.; Seabra, A. P.; Dorella, C.; Lemos, B. F. \& Pordeus, I. A. Prevalence of sleep bruxism in a group of Brazilian schoolchildren. Eur. Arch. Paediatr. Dent., 11(4):192-5, 2010.

Serra-Negra, J. M.; Ramos-Jorge, M. L.; Flores-Mendoza, C. E.; Paiva, S. M. \& Pordeus, I. A. Influence of psychosocial factors on the development of sleep bruxism among children. Int. J. Paediatr. Dent., 19(5):309-17, 2009.

Simões-Zenari, M. \& Bitar, M. L. Factors associated to bruxism in children from 4-6 years. Pro Fono, 22(4):465-72, 2010.

Varalakshmi Reddy, S.; Praveen Kumar, M.; Sravanthi, D.; Mohsin, A. H. B. \& Anuhya, V. Bruxism: A literature review. J. Int. Oral Health, 6(6):105-9, 2014.
Vieira-Andrade, R. G.; Drumond, C. L.; Martins-Júnior, P. A.; Corrêa-Faria, P.; Gonzaga, G. C.; Marques, L. S. \& Ramos-Jorge, M. L. Prevalence of sleep bruxism and associated factors in preschool children. Pediatr. Dent., 36(1):46-50, 2014.

Dirección para Correspondencia:

Dr. Héctor Sandoval

Cirujano Dentista

Universidad de Concepción

Concepción

CHILE

Email: hectorsandoval@udec.cl

Recibido: 09-11-2015

Aceptado: 10-12-2015 